\title{
Óleo de copaíba (Copaifera langsdorfii Desf.) em padrões reprodutivos de camundongos e no desenvolvimento embriofetal
}

\author{
LOURENÇO, A.C.S.; MIGUEL, L.K.; GUARIDO, K.L.; SENSIATE, L.A.; SALLES, M.J.S.* \\ Departamento de Biologia Geral, Centro de Ciências Biológicas, Universidade Estadual de Londrina, Rodovia \\ Celso Garcia Cid, Pr 445 Km 380, Caixa Postal 6001, CEP: 86055-900, Londrina-Brasil. *salmjs00@gmail.com
}

\begin{abstract}
RESUMO: A utilização de plantas medicinais sempre foi bem difundida, porém hoje se faz necessária uma abordagem científica para comprovar sua eficácia. Este estudo foi realizado para avaliar a possível toxicidade materna e teratogenicidade do óleo de copaíba, um óleo resina exudado do tronco de Copaifera langsdorfii, muito utilizado na medicina natural. Três doses de óleo de copaíba, administradas por gavage durante 5 dias do período gestacional de fêmeas de camundongos, foram testadas $0,3 \mathrm{~mL} \mathrm{Kg}^{-1}, 0,6 \mathrm{~mL} \mathrm{Kg}^{-1} \mathrm{e} 0,9 \mathrm{~mL} \mathrm{Kg}^{-1}$ (p.c.). Em relação ao ganho de peso materno, peso dos órgãos, número de fetos vivos e implantes e viabilidade fetal, não houve diferença estatística entre os grupos. Os dados demonstram que este fitoterápico não apresenta toxicidade materna. Com relação às médias de peso e comprimento fetal dos grupos tratados, houve diferença estatística quando comparados ao controle, mas os fetos ainda se encontravam dentro do peso adequado à idade de prenhez. A prole das fêmeas tratadas não apresentou malformações ou alterações externas, viscerais e esqueléticas. Os resultados deste estudo indicam que o óleo de copaíba, nas doses administradas e período estudado, não apresentou toxicidade materna ou causou teratogenicidade na prole das fêmeas tratadas. Portanto, podemos considerar seu uso seguro durante o período gestacional.
\end{abstract}

Palavras-chave: Copaifera langsdorfii, plantas medicinais, toxicidade, teratogenicidade, gestação

\begin{abstract}
Copaiba oil (Copaifera langsdorfii Desf.) on mouse reproductive patterns and embryonic or fetal development. The use of medicinal plants has always been widely spread, but today a scientific approach is needed to prove their efficiency. The present study was performed to evaluate the possible maternal toxicity and teratogenicity of copaiba oil, a resin oil exudate from the trunk of Copaifera sp., extensively used in natural medicine. Three copaiba oil levels, administered through gavage for 5 days during the gestational period of female mice, were tested: $0.3 \mathrm{~mL} \mathrm{Kg}^{-1}, 0.6 \mathrm{~mL} \mathrm{Kg}^{-1}$ and $0.9 \mathrm{~mL} \mathrm{Kg}^{-1}$ (b.w.). As regards maternal weight gain, organ weight, live fetus number, implants and fetal viability, there was no statistical difference among groups. Data indicate that this phytotherapic drug does not show maternal toxicity. Considering the means of fetal weight and length of treated groups, there was statistical difference when compared with the control group, but the fetuses were still within the appropriate weight to that pregnancy age. The offspring from treated females did not present external, visceral and skeletal alterations or malformations. The results from this study indicate that copaiba oil at the administered levels and studied period did not present maternal toxicity or cause teratogenicity to the offspring of treated females. Therefore, its use can be considered safe during pregnancy.
\end{abstract}

Key words: Copaifera langsdorfii, medicinal plants, toxicity, teratogenicity, pregnancy

\section{INTRODUÇÃO}

O uso de plantas medicinais para tratamento, cura e prevenção de doenças tem sido descrito por muitos povos desde os tempos mais remotos. Devido a esse uso, surgiram interesses comerciais e científicos e, por isso, tornou-se necessária a avaliação da eficácia e segurança dessas plantas. Para muitos, o conceito de natural significa a ausência de produtos químicos, que são aqueles que podem

Recebido para publicação em 06/11/2008

Aceito para publicação em 18/03/2009

Rev. Bras. PI. Med., Botucatu, v.11, n.4, p.407-413, 2009. 
representar perigo. Esse conceito é equivocado, já que muitas plantas contêm substâncias com ações tóxicas sobre organismos vivos. A função dessas substâncias para os produtores é de defesa contra predadores, por isso não é de surpreender que muitas plantas acumulem substâncias de elevada toxicidade (Mengue et al., 2001).

Durante a gestação, a utilização de qualquer medicamento deve ser feita com cuidado, já que algumas substâncias tóxicas podem atravessar a barreira placentária e interferir no desenvolvimento embriofetal, muitas vezes de forma negativa (Oestensen et al., 2006). Esse mesmo cuidado deve ser aplicado ao uso de plantas medicinais, principalmente devido à escassez de informações sobre as mesmas.

O óleo de copaíba é uma substância muito utilizada no contexto de medicina natural e se destaca na Amazônia e no nordeste brasileiro pelas supostas propriedades terapêuticas, em virtude da utilização para muitas enfermidades. As plantas pertencentes ao gênero Copaifera L. (Leguminosae, Caesalpinoideae) são nativas das regiões tropicais da América Latina (Dwyer, 1951). Existem diversas espécies de copaíba, de acordo com a região de ocorrência, todas muito parecidas, com as mesmas aplicações e os mesmos nomes. A copaíba é conhecida popularmente como copaíba, copaíba-vermelha, bálsamo, oleiro, copaíbada-várzea (AM), copaibeira-de-minas, copaúba, cupiúva, óleo-vermelho, pau-de-óleo (MG), podoí $(\mathrm{PI}, \mathrm{CE})$. Dessas árvores é exudado, através de furo realizado no tronco, um óleo-resina chamado óleo de copaíba. Esse óleo é transparente, com coloração variando de amarelo pálido ao castanho dourado, algumas vezes incolor, de viscosidade variável, possuindo cheiro forte e sabor amargo. Possui uma diversificada classe de substâncias, conhecida como compostos terpênicos. Já foram isolados nessas plantas o ácido copálico, ácido hardwickico e colavenol, sendo que este último possui atividade antitumoral em ratos (Ohsaki et al., 1994). As propriedades medicinais do óleo de copaíba são conhecidas pelos índios latino-americanos que provavelmente observaram que os animais se esfregavam nos troncos das copaibeiras para curar suas feridas (Barléu, 1974). De acordo com a medicina folclórica, o óleo de copaíba apresenta propriedade antiinflamatória, antitumoral e antitetânica (Lewis \& Elvin-Lewis, 1977). No Brasil seiscentista, o Padre José de Anchieta citava o óleo de copaíba como potente cicatrizante. Atualmente, esse óleo é comercializado em farmácias e lojas de produtos naturais de todo o país, com indicações diversificadas, sendo muito utilizado no contexto da medicina natural.

Dentre as várias propriedades do óleo de copaíba, as estudadas foram antiinflamatória (Basile et al., 1988), analgésica (Fernandes et al., 1992), cercaricida (Gilbert et al., 1972), repelente de insetos (Lacey et al., 1981), antitumoral (Ohsaki et al., 1994) e antimicrobial (Casamada, 1968). Em estudos realizados por Sena \& Chen (1998) foi observada a atividade anticolinesterásica do óleo em plasma de camundongos; indicando, dessa maneira, o potencial tóxico deste óleo. A presença da atividade mutagênica e tóxica do óleo de copaíba (Copaifera langsdorfii Desfon) foi demonstrada pelo teste do micronúcleo em eritrócitos policromáticos da medula óssea de camundongos em estudos feitos por Chen-Chen \& Sena (2002). Porém Veiga-Júnior et al. (2007) e Gomes et al. (2007) não observaram nenhum efeito citotóxico do óleo.

Em razão da ampla utilização empírica do óleo de copaíba e pela falta de conhecimento da toxicidade desta substância, investigamos por meio da análise do desenvolvimento intra-uterino, os possíveis efeitos indutores de alterações em animais submetidos a tratamento e na prole.

\section{MATERIAL E MÉTODO}

\section{Animais}

Foram utilizados camundongos (Mus musculus), machos e fêmeas adultos, da linhagem Swiss, provenientes do Biotério Central do Centro de Ciências Biológicas da Universidade Estadual de Londrina. Os animais passaram por período de aclimatação de sete dias no biotério setorial, foram mantidos em gaiolas de polipropileno com tampa de arame zincado, contendo maravalha, com água e ração ad libitum, em regime de luminosidade e temperatura controlada (ciclo de 12 horas claro/ escuro, a $22 \pm 2^{\circ} \mathrm{C}$ ). Todo o experimento foi aprovado pelo Comitê de Ética em Experimentação Animal da Universidade Estadual de Londrina.

\section{Delineamento experimental}

Sessenta fêmeas foram distribuídas aleatoriamente em 4 grupos experimentais, cada um contendo 15 animais. Três grupos de animais receberam via gavage óleo de copaíba $(\mathrm{OC})$ da marca Vid'Amazon, extraído de Copaifera sp. Cada grupo foi tratado com uma das três concentrações do óleo: OC 0,3 com 0,3 mL Kg ${ }^{-1}$ (p.c.); OC 0,6 com 0,6 mL $\mathrm{Kg}^{-1}$ (p.c.) e OC 0,9 com 0,9 mL Kg-1 (p.c.) no 8, 9, 10 , 110 e 12 o dias de gestação, período de organogênese da espécie. Os animais do grupo controle receberam triglicerol de cadeia média (TCM), utilizado para diluição do óleo de copaíba sob o mesmo delineamento experimental. Durante a gestação, as doses de óleo de copaíba administradas foram baseadas no peso da fêmea no dia do tratamento. O TCM foi escolhido por ser um lipídio com característica inerte e normalmente utilizado 
como suplemento alimentar em diversas espécies animais. A concentração intermediária $\left(0,6 \mathrm{~mL} \mathrm{Kg}^{-1}\right)$ foi encontrada na literatura consultada (Araújo Júnior et al., 2005), sendo então escolhidos os valores das doses inferior e superior.

Os animais foram acasalados e o dia em que se verificou o "plug" vaginal foi designado dia zero de prenhez. Para avaliação do ganho de peso materno, as fêmeas foram pesadas durante todo o período de prenhez. No 18 dia, foram eutanasiadas por deslocamento cervical e, em seguida, foi realizada a laparotomia. O útero com fetos foi pesado e realizouse a histerectomia e onfalectomia. A correção do ganho de peso materno foi feita considerando-se o ganho de peso materno menos peso do útero gravídico. O conteúdo uterino das fêmeas foi analisado, verificando-se a presença de reabsorções, número de fetos vivos e mortos, peso fetal e placentário, comprimento fetal, e sob microscópio estereoscópio foi feita a análise sistemática para detecção de possíveis malformações estruturais externas. O número de sítios de implantação foi determinado pelo método de Salewski (1964). Com estes dados foram calculadas taxa de perdas pósimplantação e taxa de viabilidade fetal. A adequação do peso fetal à idade de prenhez foi determinada baseando-se no método estabelecido por Calderon (1992). Os fetos presentes na faixa entre a média do controle, mais ou menos o desvio padrão, vezes o fator de correção, são os fetos que apresentam peso adequado para a idade de prenhez (AIP). Aqueles que se encontram abaixo dessa faixa são os fetos com peso inferior ao peso adequado à idade de prenhez (PIP). Os com peso acima dessa faixa são considerados fetos com peso acima do peso adequado para a idade de prenhez (GIP). A metade dos fetos foi fixada na mistura de Bodian para a análise visceral, enquanto a outra metade foi fixada em acetona e submetida à técnica de Alizarina red descrita por Staples \& Schnell (1964), para a realização da análise esquelética.

A análise visceral foi conduzida através da combinação de cortes/microdissecção proposta por Barrow \&Taylor (1969) para estudo de tórax e abdome e pelos cortes estratégicos propostos por Wilson (1965) para estudo da cabeça. Os fetos submetidos à análise esquelética foram avaliados para detecção de anomalias do crânio, esterno, vértebras, costelas, bacia, clavícula, falanges, metacarpo e metatarso segundo o método de Taylor (1986). Estas avaliações foram executadas sob microscópio estereoscópio.

\section{Análise Estatística}

Os dados absolutos com distribuição normal foram analisados pela ANOVA (Análise de variância), complementada com o teste de Tukey ou através do teste t de Student; e os com distribuição não-normal foram analisados por Kruskal-Wallis, complementado com o teste de Dunn ou pelo teste Mann - Whitney. O nível de significância considerado foi de 5\%.

\section{RESULTADO}

Dentre os parâmetros analisados para verificar a toxicidade do óleo de copaíba sobre a performance reprodutiva das fêmeas, não houve diferença estatística entre os grupos em relação a ganho de peso materno corrigido, peso relativo dos órgãos maternos, número de implantes e de fetos vivos e, ainda, viabilidade fetal ou perda pósimplantacional, apresentado na Tabela 1. Com relação ao desenvolvimento intra-uterino dos fetos, houve diferença estatisticamente significativa, principalmente nos valores médios de peso e comprimento fetal. Observa-se, através das médias apresentadas na Tabela 2, que na dose mais baixa de óleo de copaíba $\left(0,3 \mathrm{~mL} \mathrm{Kg}^{-1}\right)$ os fetos apresentaram-se com peso e comprimento maior do que o grupo controle, enquanto as outras doses de óleo de copaíba proporcionaram uma diminuição no peso e comprimento fetais. No entanto, quando realizada a adequação individual dos pesos fetais de cada grupo, pôde-se observar que, mesmo suas médias tendo diferenciado do controle, a maior parte de seus fetos encontrava-se com peso adequado à idade de prenhez, mostrado na Figura 1. Não houve diferença estatística entre as médias de pesos das placentas nem entre índices placentários dos grupos (Tabela 2).

Quanto a alterações externas, foi encontrado apenas um feto com anoftalmia unilateral. Nesse estudo, a exposição materna ao óleo de copaíba não influenciou na formação esquelética e nem na ossificação dos 248 fetos analisados (Tabela 3). Dados relativos a outros ossos não foram apresentados em tabela por não haver alterações nos mesmos. Malformações viscerais não foram observadas em nenhum grupo experimental. Foram avaliados 203 fetos.

\section{DISCUSSÃO}

Na terapia moderna, observa-se crescente redescoberta do valor das plantas medicinais para a cura, prevenção e tratamento de doenças. Apesar disso, o uso incorreto desses fitoterápicos pode causar danos desastrosos à saúde do individuo. A literatura é escassa com relação a estudos envolvendo propriedades e toxicidade de plantas medicinais.

Comparado com estudos disponíveis na literatura, este trabalho inova por apresentar delineamento experimental inédito, o que dificulta a comparação com outros estudos com a Copaifera langsdorfii. No entanto, plantas que pertencem aos 
TABELA 1. Efeito do tratamento com óleo de copaíba na performance reprodutiva de camundongos fêmeas.

\begin{tabular}{|c|c|c|c|c|}
\hline & TCM & OC0,3 & OC0,6 & OC0,9 \\
\hline № de fêmeas prenhes & 15 & 15 & 15 & 15 \\
\hline Ganho de peso materno (g) & $19,132 \pm 2,067$ & $17,001 \pm 1,152$ & $17,379 \pm 2,028$ & $20,720 \pm 1,621$ \\
\hline Peso do útero gravídico (g) & $12,570 \pm 1,912$ & $11,709 \pm 1,078$ & $14,078 \pm 1,688$ & $14,208 \pm 1,529$ \\
\hline Ganho de peso materno corrigido (g) & $6,562 \pm 0,6648$ & $5,291 \pm 0,5700$ & $3,301 \pm 0,8650$ & $6,512 \pm 0,8327$ \\
\hline Peso do coração materno (g) & $0,1649 \pm 0,0057$ & $0,1739 \pm 0,0075$ & $0,1698 \pm 0,0096$ & $0,1743 \pm 0,0062$ \\
\hline Peso dos pulmões materno $(\mathrm{g})$ & $0,2142 \pm 0,0099$ & $0,2390 \pm 0,0066$ & $0,2150 \pm 0,0104$ & $0,2122 \pm 0,0085$ \\
\hline Peso dos rins materno $(\mathrm{g})$ & $0,3579 \pm 0,0176$ & $0,3333 \pm 0,0147$ & $0,3517 \pm 0,01379$ & $0,3985 \pm 0,01647$ \\
\hline Peso do fígado materno $(\mathrm{g})$ & $2,141 \pm 0,1037$ & $2,168 \pm 0,0959$ & $2,078 \pm 0,1274$ & $2,344 \pm 0,0509$ \\
\hline Número de implantações & $9,133 \pm 1,257$ & $9,733 \pm 0,6652$ & $9,800 \pm 1,105$ & $11,400 \pm 0,7224$ \\
\hline Número de fetos vivos & $7,267 \pm 1,221$ & $5,867 \pm 0,6315$ & $8,400 \pm 1,086$ & $8,533 \pm 1,046$ \\
\hline Viabilidade fetal (\%) & $79,97 \pm 6,910$ & $62,86 \pm 6,664$ & $86,98 \pm 4,924$ & $72,11 \pm 6,009$ \\
\hline Perda pós implantacional (\%) & $20,03 \pm 6,910$ & $27,14 \pm 6,664$ & $13,02 \pm 4,924$ & $27,89 \pm 6,009$ \\
\hline
\end{tabular}

Dados representados em média \pm SEM. Médias seguidas de * são estatisticamente diferentes do grupo controle. TCM: grupo controle. OC: grupos tratados (seguidos das doses correspondentes ao tratamento). ANOVA, $p=0,05$.

TABELA2. Efeito do tratamento com óleo de copaíba no desenvolvimento intra-uterino dos fetos.

\begin{tabular}{lcccc}
\hline & TCM & OC0,3 & OC0,6 & OC0,9 \\
\hline Peso fetal individual $(\mathrm{g})$ & $1,360 \pm 0,0141$ & $1,488 \pm 0,0122^{*}$ & $1,309 \pm 0,0166^{*}$ & $1,236 \pm 0,0185^{\star}$ \\
Comprimento fetal $(\mathrm{cm})$ & $2,495 \pm 0,0119$ & $2,526 \pm 0,0140^{*}$ & $2,407 \pm 0,0166^{*}$ & $2,446 \pm 0,0135^{\star}$ \\
Peso placentário individual $(\mathrm{g})$ & $0,1064 \pm 0,0023$ & $0,1271 \pm 0,0029$ & $0,1013 \pm 0,0017$ & $0,1026 \pm 0,0022$ \\
Índice placentário & $0,0787 \pm 0,0017$ & $0,0863 \pm 0,0023$ & $0,0784 \pm 0,0014$ & $0,8624 \pm 0,0014$
\end{tabular}

Dados representados em média \pm SEM. Médias seguidas de * são estatisticamente diferentes do grupo controle. TCM: grupo controle. OC: grupos tratados (seguidos das doses correspondentes ao tratamento). ANOVA, $p=$ 0,05 , Kruskal-Wallis, $p=0,05$.

gêneros Copaifera L., Mikania Willd., Annona L. e Xylopia L., são ricas em ácido kaurenóico, um diterpeno com propriedades antiinflamatórias, podendo ser ele o responsável por esse efeito (Cavalcanti et al., 2006), o que permite comparação entre as mesmas.
O ganho de peso materno e o peso relativo dos órgãos não sofreram alterações causadas pelo tratamento com óleo de copaíba, portanto, fica claro que este fitoterápico não apresenta toxicidade para as fêmeas tratadas, pois a alteração desses 


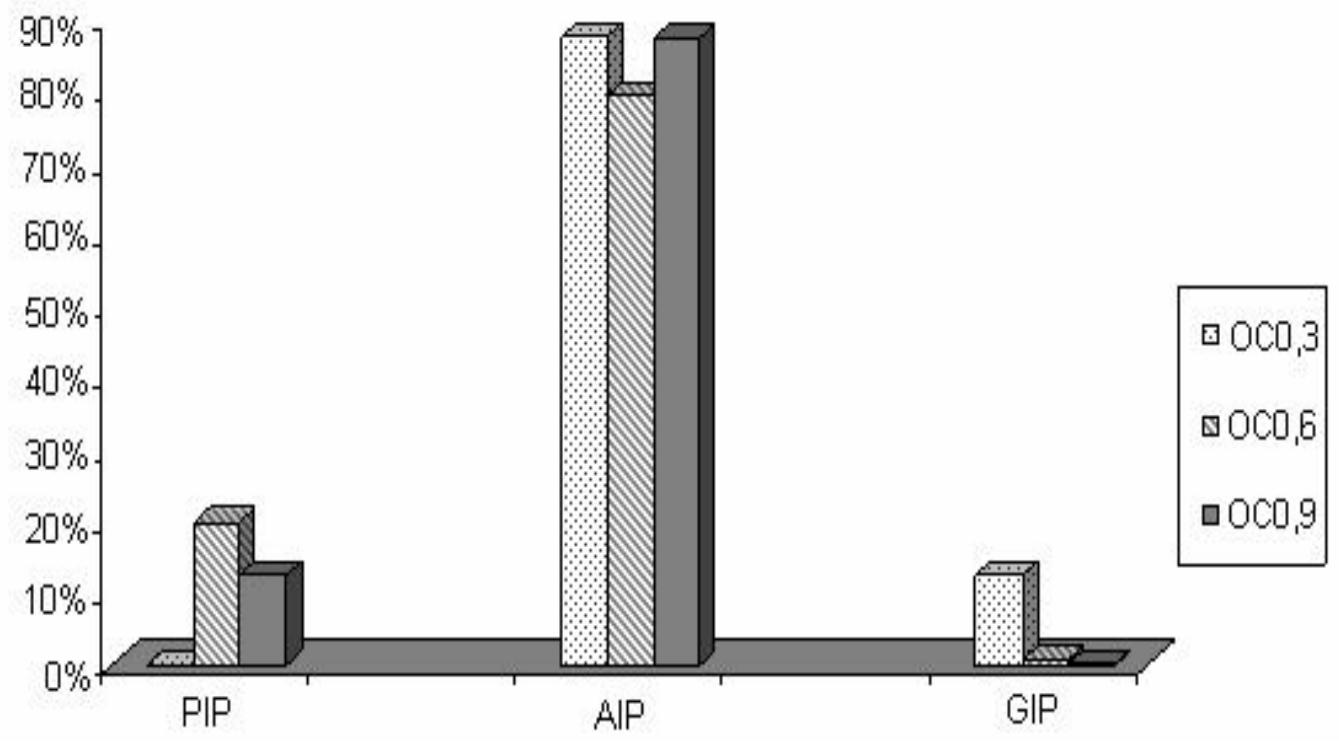

FIGURA 1. Adequação do peso fetal à idade de prenhez dos grupos tratados com óleo de copaíba. PIP: peso inferior ao peso adequado à idade de prenhez; AIP: peso adequado à idade de prenhez e GIP: peso acima do peso adequado à idade de prenhez.

TABELA 3. Número de centros de ossificação da prole de fêmeas tratadas com óleo de copaíba.

\begin{tabular}{|c|c|c|c|c|}
\hline & TCM & OC0,3 & OC0,6 & OC0,9 \\
\hline $\begin{array}{l}\text { Metacarpos em ambas } \\
\text { patas dianteiras }\end{array}$ & $3,966 \pm 0,0238$ & $3,958 \pm 0,0292$ & $3,931 \pm 0,0302$ & $3,942 \pm 0,0283$ \\
\hline $\begin{array}{l}\text { Primeiras falanges em } \\
\text { ambas patas dianteiras }\end{array}$ & $3,983 \pm 0,0169$ & $3,979 \pm 0,0208$ & $3,972 \pm 0,0195$ & $4,000 \pm 0,000$ \\
\hline $\begin{array}{l}\text { Segundas falanges em } \\
\text { ambas patas dianteiras }\end{array}$ & $3,000 \pm 0,000$ & $3,000 \pm 0,000$ & $3,000 \pm 0,000$ & $3,000 \pm 0,000$ \\
\hline $\begin{array}{l}\text { Metatarsos em ambas } \\
\text { patas traseiras }\end{array}$ & $5,000 \pm 0,000$ & $5,000 \pm 0,000$ & $5,000 \pm 0,000$ & $5,000 \pm 0,000$ \\
\hline $\begin{array}{l}\text { Primeiras falanges em } \\
\text { ambas patas traseiras }\end{array}$ & $4,814 \pm 0,0511$ & $4,833 \pm 0,0544$ & $4,819 \pm 0,0457$ & $4,812 \pm 0,0474$ \\
\hline $\begin{array}{l}\text { Segundas falanges em } \\
\text { ambas patas traseiras }\end{array}$ & $1,746 \pm 0,0572$ & $1,792 \pm 0,0592$ & $1,806 \pm 0,0469$ & $1,739 \pm 0,0533$ \\
\hline Vértebras caudais & $7,136 \pm 0,0449$ & $7,104 \pm 0,0446$ & $7,069 \pm 0,0302$ & $7,101 \pm 0,0366$ \\
\hline
\end{tabular}

Dados representados em média \pm SEM. Médias seguidas de * são estatisticamente diferentes do grupo controle. TCM: grupo controle. OC: grupos tratados (seguidos das doses correspondentes ao tratamento). Kruskal-Wallis, $p=0,05$.

parâmetros é um indicador de toxicidade materna. Estudos com Annona squamosa, realizados por Damasceno et al. (2002a), apresentaram resultados semelhantes, em que a planta testada também não apresentou alterações nos parâmetros de toxicidade materna.

A interpretação das alterações nas taxas reprodutivas pode identificar o período no qual os efeitos tóxicos reprodutivos se estabeleceram. Observou-se que não houve diferença estatística significativa entre taxas de reabsorção e perdas pósimplantacionais, assim como entre a média do número de implantações e de fetos vivos. Os dados demonstram que o óleo de copaíba não apresentou efeito embrioletal. Esses resultados estão em concordância com os obtidos por Damasceno et al. 
(2002a), que investigaram os efeitos de Annona squamosa sobre a gestação de ratas, e por Domaracký et al. (2007), que estudou os efeitos de óleos essenciais antiinflamatórios sobre a préimplantação e desenvolvimento de camundongos.

No presente estudo, o óleo de copaíba causou alteração significativa em peso e comprimentos fetais, mesmo que os fetos ainda estivessem dentro do peso adequado para a idade de prenhez. No entanto, segundo Aliverti et al. (1979), o peso fetal não é parâmetro conclusivo em estudos de desenvolvimento porque pode variar dependendo do tamanho da ninhada. Por isso a determinação de centros de ossificação foi usada neste trabalho como parâmetro adicional para avaliar o desenvolvimento fetal. Como a exposição materna ao óleo de copaíba não influenciou na ossificação, confirma-se que este componente não altera o desenvolvimento intra-uterino e não causa efeitos teratogênicos. Esses dados estão em coerência com estudos realizados sobre o efeito de antiinflamatórios no desenvolvimento. O Ácido acetilsalicílico não causa influência na ossificação da prole de ratas tratadas com $50 \mathrm{~g} \mathrm{Kg} \mathrm{K}^{-1}$ (Damasceno et al., 2002b). Dados sobre outras plantas com efeito antinflamatório, que tiveram a toxicidade sobre a prole de mamíferos não foram encontrados na literatura.

Muitos teratologistas preconizam que substâncias químicas que passam da circulação materna para os fluidos uterinos e penetram no embrião no período de pré-implantação podem causar a morte do embrião ou a sobrevivência sem danos, porque, nesta situação, células tronco têm a capacidade de substituir células danificadas, resultando em desenvolvimento embrionário normal (Carlson, 1996). No entanto, teratógenos que passam barreira placentária podem não ser letais ao embrião, mas podem causar desvios de desenvolvimento, como malformação congênita, retardo do crescimento, déficit funcional e comportamental. Sesquiterpenos presentes na composição do óleo de copaíba atravessam a membrana placentária, e já foram identificados em plantas tóxicas como Dittrichia graveolens (Lanzetta et al., 1991). Enfatiza-se que a administração do óleo de copaíba nas doses e período estudado não apresentou toxicidade materna ou causou teratogenicidade na prole das fêmeas tratadas, podendo ser considerado seguro o seu consumo durante o período de gestação.

\section{REFERÊNCIA}

ALIVERTI, V. et al. The extent of fetal ossification as an index of delayed development in teratogenic studies on the rat. Teratology, v.20, p.237-42, 1979.

ARAÚJO JÚNIOR, F.A.; BRAZ, M.N.; ROCHA NETO, G. Efeito do óleo de copaíba nas aminotransferases de ratos submetidos à isquemia e reperfusão hepática com e sem pré-condicionamento isquêmico. Acta Cirúrgica Brasileira, v.20, p.93-9, 2005.

BARLÉU, G. História dos feitos recentes praticados durante 20 anos no Brasil. São Paulo: Universidade de São Paulo, 1974. 141p. (Coleção Reconquista do Brasil, v.15.)

BARROW, M.V.; TAYLOR, W.J. A rapid method for detecting malformations in rat fetuses. Journal of Morphology, v.127, p.291-306, 1969.

BASILE, A.C.; SERTIE, J.A.; FREITAS, P.C.D. Antiinflammatory activity of oleoresin from Brazilian copaiba. Journal of Ethnopharmacology, v.22, p.101- 9, 1988.

CALDERON, I.M.P. et al. Diabete e gravidez experimental em ratas: 1. Indução do diabete, obtenção e evolução da prenhez. Acta Cirúrgica Brasileira, v.7, p.142-6, 1992. CASAMADA, R.S.M. Farmacognosia con farmacodinamia. Barcelona: Científico Médica,1968. 1121p.

CARLSON, B.M. Embriologia humana e biologia do desenvolvimento. Rio de Janeiro: Guanabara-Koogan, 1996. $424 p$.

CAVALCANTI, B.C. et al. Genotoxicity evaluation of kaurenoic acid, a bioactive diterpenoid present in copaiba oil. Food and Chemical Toxicology, v.44, p.388-92, 2006. CHEN-CHEN, L.; SENA, M.A. Atividade tóxica e mutagênica do óleo de copaíba (Copaifera langsdorfii) em camundongos. Revista Brasileira de Plantas Medicinais, v.5, p.37-40, 2002.

DAMASCENO, D.C. et al. Effects of Annona squamosa extract on early pregnancy in rats. Phytomedicine, v.9, p.667-72, 2002a.

DAMASCENO, D.C. et al. Efeito do ácido acetilsalicílico na performance reprodutiva e na prole de ratas Wistar. Revista da Associação Médica Brasileira, v.48, p.3126, 2002b.

DOMARACKY, M. et al. Effects of selected plant essential oil on the growth and development of mouse preimplantation embryos in vivo. Physiological Research, v.56, p.97-104, 2007.

DWYER, J.D. The Central American, west Indian and South American species of Copaifera L. (Caesalpiniaceae). Brittonia, v.7, p.143-72, 1951.

FERNANDES, R.M.; PEREIRA, N.A.; PAULO, L.G. Antiinflammatory activity of copaíba balsam (Copaifera cearensis, Huber). Revista Brasileira de Farmácia, v.73, p.53-6, 1992.

GILBERT, B. et al. A atividade anti-helmíntica de óleos essenciais e de seus componentes químicos. Anais da Academia Brasileira de Ciências, v.44, p.423-8, 1972. GOMES, N.M. et al. Antinociceptive activity of Amazonian copaiba oils. Journal of Ethnopharmacology, v.109, p.486-92, 2007.

LACEY, L.A.; SCHRECK, C.E.; MCGOVERN, T.P. Native and experimental repellent against black flies in the Amazon basis of Brazil. Mos News, v.41, p.376-9, 1981. LANZETTA, R. et al. Ichthyotoxic sesquiterpenes and xanthanolides from Dittrichia-Graveolens. Phytochemestry, v.30, p.1121-4, 1991.

LEWIS, W.H.; ELVIN-LEWIS, P.F. Medical Botany: plants affecting Man's health. New York: John Willey Sons, 1977. 832p.

MENGUE, S.S. et al. Uso de plantas medicinais na gravidez. Revista Brasileira de Farmacognosia, v.11, p.21-35, 2001. 
OHSAKI, A. et al. The isolation and in vivo potent antitumor activity of clerodane diterpenoid from de oleoresin of the Brazilian medicinal plant, copaiba. Bioorganic and Medicinal Chemistry Letters, v.4, p.2889-92, 1994.

OESTENSEN, $M$. et al. Anti-inflammatory and immunosuppressive drugs and reproduction. Arthritis Research \& Therapy, v.8, p.209-27, 2006.

SALEWSKI, E. Färbemethoden zum makroskopischen Nachweis von Implantationsstellen am Uterus der Ratte. Naunyn-Schmiedeberg's Archives of Pharmacology, v.247, p.367, 1964.

SENA, M.A.; CHEN, L.C. Avaliação da atividade anticolinesterase do óleo de copaíba em plasmas de camundongos. In: SIMPÓSIO DE PLANTAS MEDICINAIS DO BRASIL, 15., 1998, Águas de Lindóia. Livro de Resumos... Águas de Lindóia: IBPM, 1998. p.173.
STAPLES, R.E.; SCHNELL, V.L. Refinements in rapid clearing technic in the $\mathrm{KOH}$-Alizarin red $\mathrm{S}$ method for fetal bone. Stain Technology, v.39, p.61-3, 1964.

TAYLOR, P. Practical teratology. London: Academic Press, 1986. 170p.

VEIGA JUNIOR, V.F. et al. Chemical composition and antiinflammatory activity of copaiba oils from Copaifera cearensis Huber ex Ducke, Copaifera reticulata Ducke and Copaifera multijuga Hayne - A comparative study. Journal of Ethnopharmacology, v.112, p.248-54, 2007. WILSON, J.G. Methods for administering agents and detecting malformations in experimental animals. In: WILSON, J.G.; WARKANY, J. (Eds.). Teratology: principals and techniques. Chicago: University of Chicago Press, 1965. p.262-77. 